anbiances Environnement sensible, architecture et espace urbain

\title{
La résistance du sensible, avenir de l'anthropologie sensorielle
}

A propos de : Joël Candau, Paul-Louis Colon (dir.), Olivier Féraud, MarieLuce Gélard, Arnaud Halloy, Tim Ingold, Anthony Pecqueux, Véronique Servais, Jean-Paul Thibaud, Bénédicte de Villers, Olivier Wathelet, Ethnographier les sens, Pétra, 2014, 337 p.

\section{Anne Bossé}

\section{OpenEdition}

\section{Journals}

Édition électronique

URL : http://journals.openedition.org/ambiances/682

DOI : $10.4000 /$ ambiances.682

ISSN : 2266-839X

Éditeur :

Direction Générale des Patrimoines - DAPA - MCC, UMR 1563 - Ambiances Architectures Urbanités (AAU)

\section{Référence électronique}

Anne Bossé, «La résistance du sensible, avenir de l'anthropologie sensorielle », Ambiances [En ligne], Comptes-rendus, mis en ligne le 19 décembre 2015, consulté le 22 septembre 2020. URL : http:// journals.openedition.org/ambiances/682 ; DOI : https://doi.org/10.4000/ambiances.682

Ce document a été généré automatiquement le 22 septembre 2020.

\section{(c) (i) $९$}

Ambiances is licensed under a Creative Commons Attribution-NonCommercial-NoDerivatives 4.0 International License. 


\section{La résistance du sensible, avenir de l'anthropologie sensorielle}

A propos de : Joël Candau, Paul-Louis Colon (dir.), Olivier Féraud, MarieLuce Gélard, Arnaud Halloy, Tim Ingold, Anthony Pecqueux, Véronique Servais, Jean-Paul Thibaud, Bénédicte de Villers, Olivier Wathelet, Ethnographier les sens, Pétra, 2014, 337 p.

\section{Anne Bossé}

\section{RÉFÉRENCE}

Joël Candau, Paul-Louis Colon (dir.), Olivier Féraud, Marie-Luce Gélard, Arnaud Halloy, Tim Ingold, Anthony Pecqueux, Véronique Servais, Jean-Paul Thibaud, Bénédicte de Villers, Olivier Wathelet, Ethnographier les sens, Pétra, 2014, 337 p.

1 Qu'y a-t-il de commun entre les phénomènes météorologiques, un quartier napolitain, les rencontres avec des dauphins et l'activité culinaire ? Il est possible, pour chacun de ces terrains de recherche, de mettre au centre la question de l'expérience sensorielle. Sous le titre «Ethnographier les sens » cet ouvrage réunit onze contributions sur des objets variés donc, mais toutes ont pour visée de prendre au sérieux les questions épistémologiques et méthodologiques que ce titre implique. Ethnographier les sens conduit alors autant à interroger la théorie de la connaissance du sujet individuel que l'anthropologie et son projet comparatif des univers sensoriels culturels, à réfléchir aux bases conceptuelles pour adosser la recherche sur les sens d'un point de vue social et culturel qu'argumenter en faveur de l'ethnographie comme méthode.

Issu d'un colloque qui s'est tenu à Liège et à Bruxelles en 2010 dans l'objectif de donner une visibilité à la recherche francophone sur les sens et de créer un dialogue avec l'anthropologie sensorielle anglo-saxonne, cet ouvrage se propose de montrer la diffusion de cette entrée par les sens dans les recherches contemporaines en anthropologie et en sociologie. Au-delà des résultats sur la perception sensorielle ou sur les usages des sens, ce sont les sens comme intermédiaire conceptuel (p. 17), à 
même d'ouvrir des pistes de recherche nouvelles et de faire bouger les lignes disciplinaires, que souhaite mettre en avant cet ouvrage.

La diversité des objets décrits mais aussi des approches des auteurs, convoquant la philosophie, la phénoménologie, le pragmatisme, l'interactionnisme ou le cognitivisme peut dérouter. Peut-être faut-il avoir déjà une idée du paysage des questionnements reliés aux sens pour mettre à profit les contributions? Même si le parti pris ethnographique, au cœur de la pratique de ces chercheurs peut intéresser nombre de praticiens des sciences sociales, y compris ceux qui ne se donnent pas comme horizon de travailler sur les sens. C'est par cet aspect que l'ouvrage est le plus percutant. Les enquêtes fouillées et l'effort de précision théorique plaident pour l'argument défendu : la question des sens est toujours déjà là. L'intégrer à son propre questionnement permet au chercheur ethnographe de ne pas user de certaines " entourloupes », au sens où les processus de catégorisations, les représentations sociales ou les expériences d'altérité doivent s'incarner et non être déclarées dans leurs modalités ou dans leurs effets.

\section{Clarifier le rôle des sens : un débat toujours actif}

4 La première partie "approcher les sens " réunit trois contributions, de Tim Ingold, Anthony Pecqueux et Paul-Louis Colon, qui s'attachent de manière féconde à des points de théorie essentiels du questionnement sur les sens: l'expérience du sentir et l'expérience perceptive. Le modèle cartésien de la vision humaine et la conception représentationnelle des sens peuvent nous sembler évidemment dépassés, pourtant les auteurs en montrent les survivances au travers de présupposés sur l'expérience de l'homme et de son environnement. Si on voit des choses et non la lumière, à l'inverse du son qui semble traverser les oreilles pour atteindre l'âme, n'est-ce pas parce que les yeux arrêtent la lumière étant donné que c'est l'esprit qui réalise l'image intérieure? Voici comment Ingold entame sa réflexion. La coupure récurrente d'un observateur comme sujet et de l'environnement comme objet à percevoir, est chaque fois remise en cause par ces auteurs. Ce que fait Ingold en instruisant que pour voir nous devons être plongés dans la lumière. Percevoir l'état de l'atmosphère est un mode d'être, à la différence de la perception du paysage qui est un mode d'observation. Les conditions atmosphériques sont ce par quoi nous percevons, et non l'objet de notre perception; le vent, le tonnerre sont des expériences du ressentir. Même intérêt pour mieux préciser l'expérience du sentir dans le texte de Paul-Louis Colon pour pouvoir répondre à la controverse sur l'ethnocentrisme des études sur les sens. L'individu ne prélève pas de l'information à propos du monde. En reprenant les travaux de Erwin Straus (psychiatre allemand), un constat s'impose : le sentir n'est pas une forme de connaissance, il est mouvement, expérience unitaire et continue. Si le sentir est ce rapport immédiat au monde et aux autres, la perception est le rapport réflexif que l'individu endosse face à sa propre situation. Les sensations sont ainsi le produit de la perception définie comme une différenciation progressive opérée par le sujet pris dans sa relation totale au monde. Aussi il existe un partage universel d'un sentir et des systèmes perceptifs culturels qui découpent, avec des variations, dans cette expérience. Poursuivant ce questionnement, Anthony Pecqueux s'attache à l'expérience de la perception. L'enjeu de sa réflexion se situe à nouveau sur les intermédiaires entre perception et chose perçue chers au discours des sciences cognitives avec leur goût des illusions 
perceptives. L'auteur suit la théorie de la perception à partir du langage descriptif de l'homme de la rue de J. Austin qui conclut à la «fiabilité ordinaire des apparences ». Avec des exemples d'enquêtes ethnographiques et les travaux des ethnométhodologues, l'auteur montre que la perception n'est pas le résultat de processus cognitifs, elle est plus directe, du fait de l'habitude, grâce à l'acquisition progressive de concepts, du fait de la confiance également. Autant d'aspects de la dimension sociale de la perception qui ressortent strictement du perceptif et non du conceptuel.

5 La part cognitive de l'individu se trouve finalement revue à la baisse, replacée dans une expérience de l'être vivant et repensée dans les relations engagées avec le monde : « les sens ne véhiculent plus des modes de pensée ", «ils ne sont plus compris comme des canaux qui altèrent l'information sur soi et le monde qu'ils transportent, mais comme des formes d'être avec le monde, qui préexistent à toute pensée du sujet et de l'objet » conclut Paul-Louis Colon (p. 96).

\section{De la diversité des sujets sensibles}

6 La seconde partie de l'ouvrage regroupe quatre textes appuyés par des enquêtes ethnographiques et qui s'attachent aux usages des sens. Marie-Luce Gélard analyse les « langages des sens » et les expressions corporelles et sensorielles des femmes dans une région du Sahara du sud-est marocain. Olivier Féraud renseigne les usages de la voix et l'environnement sonore d'un quartier populaire au centre de Naples. Olivier Wathelet décrit les compétences perceptives dans le cadre de l'activité culinaire, et Bénédicte De Villers, enfin, se saisit de la peur dans les relations hommes chiens pour l'étudier comme un agencement sensoriel spécifique. Chaque texte s'inscrit dans un rapport à la question des sens différent: valoriser les manifestations non verbales, montrer comment les relations sociales sont liées à des productions sensibles, qualifier l'activité perceptive d'experts ou montrer les liens entre émotions et organisation sensorielle. Usage différencié de chacun des sens, revendication d'une approche sensible et non seulement technique (du sonore), valorisation de la cognition située et de l'agir avec (une matérialité changeante dans le cas de l'activité culinaire), réflexion sur l'expérience de la mise en présence: les enjeux théoriques ne se raccrochent pas toujours aux éléments conceptuels posés dans les premiers textes, certains étant parfois déjà plus défrichés. Chaque fois par contre l'approche ethnographique - avec dans certains cas des enquêtes très longues - produit des descriptions singulières à même de participer d'une exigence scientifique importante, celle d'un langage qui traduit les inventions sensorielles humaines: on y découvre l'odeur sèche, la voix haute, le son du brûlé ou encore le rôle de certaines odeurs dans la peur.

7 Dans la troisième partie intitulée "ouvertures sensorielles", trois derniers textes ouvrent vers des horizons très contrastés, de la question des sens et de l'identité à celle des ambiances et à l'expérience de l'enchantement. Le texte de Joël Candau cherche à faire valoir comment de nombreux stimuli sensoriels contribuent à forger nos représentations identitaires qui ne relèvent pas que de mécanismes conscients et maitrisés. Avec une approche qui s'alimente des travaux en contexte expérimental des neurosciences, l'auteur s'attache notamment à montrer comment ce qu'il nomme «l'essentialisme sensoriel» - une caractéristique humaine ici entendue comme universelle - est engagé dans les rapports entre individus de couleurs différentes. Il 
souhaite ainsi prendre en défaut les théories de l'identité qui la font valoir comme une construction en cherchant à écarter la part biologique qui racialise les rapports sociaux, et que pourtant le sens commun rend selon lui évidente. La démonstration peut laisser perplexe, de notre côté on aimerait plutôt comprendre ce que les individus font des émotions suscitées par des interactions Noir/Blanc que s'interroger sur l'activation de l'amygdale à la vue de sujets de couleurs différentes de soi. En écho, l'ouvrage de Florence Weber, qui retrace l'histoire de l'anthropologie et montre comment cette discipline doit à plusieurs reprises se situer par rapport aux théories biologiques et à « la pente naturelle de notre esprit » comme le dit Joel Candau, est une piqure de rappel intéressante : " le racisme indigène ne doit pas être combattu par des arguments scientifiques, il doit être analysé comme un fait social » (Weber, 2015).

8 Le texte de Jean-Paul Thibaud emmène lui sur bien d'autres pistes de réflexion. Sa forme même est reliée au questionnement sur le sensible - pour que la pensée urbaine se fasse sensibilité atmosphérique. L'auteur explore six notions sonores pour rendre sensible les territoires contemporains et leurs devenirs. Tonalité, médium, résonance, coalescence, performance, entretien et imprégnation tentent de dire autrement l'expressivité des espaces urbains, territoire avant tout d'habitabilité. Le dernier texte enfin est un travail de montée en généralité théorique sur l'expérience de l'enchantement grâce au croisement de deux travaux distincts, l'un de Arnaud Halloy sur la possession dans le culte Xangô de Récife, l'autre de Véronique Servais sur « la rencontre dauphin». Par comparaison, ils identifient les conditions nécessaires à l'enchantement, ses différentes étapes, en insistant sur l'importance de la description de ce dispositif, où le sensoriel est convoqué dans l'apprentissage, dans l'expérience corporelle, mais aussi dans l'environnement comme agencement.

9 Le choix de l'exhaustivité pour cette note de lecture cherche à se conformer au souci initial de l'ouvrage, rendre compte d'une diversité de thématiques et de perspectives théoriques qui en fait, en effet, la valeur. Si chacun des textes trouvera évidemment plus ou moins d'intérêt aux yeux de lecteurs recherchant moins un panorama, on retiendra avant tout le propos global : l'incontournable expérience sensorielle dans les questions de sociétés. Le développement des sciences cognitives ou la socialisation des sciences de la nature emblématisée par l'Anthropocène obligent les sciences humaines et sociales à faire valoir leurs travaux déjà anciens sur les sens et à réaffirmer leurs prérogatives sur l'homme comme être vivant et sur l'expérience sensorielle et ce qu'elle fait à l'individu social. Peut-être la difficulté est-elle que la pensée scientifique de l'expérience sensorielle, de fait, la rend peu triviale, alors même que plusieurs textes soulignent justement à quel point c'est là aussi, dans le sentir non senti que se loge une commune humanité. Le chercheur, engagé souvent du côté de la rationalité peine à jouer son rôle quand le cognitif s'absente... C'est ce que rappelle malicieusement l'ouvrage La vie, mode mineur: "si la vie était telle que la décrivent le plus souvent les sciences sociales, elle serait sans nul doute invivable » (Rémy, Denizeau, 2015). 


\section{BIBLIOGRAPHIE}

Rémy, Catherine; Denizeau, Laurent (dir.). 2015. La vie, mode mineur. Paris : Presses des Mines.

Weber, Florence. 2015. Brève histoire de l'anthropologie. Paris : Flammarion.

\section{AUTEURS}

\section{ANNE BOSSÉ}

Anne Bossé est enseignante en urbanisme et projet urbain à l'Ecole Nationale Supérieure d'Architecture de Paris Malaquais et chercheure au CRENAU (UMR CNRS 1563 Ambiances, Architectures, Urbanités / ENSA Nantes). Architecte de formation et docteure en géographie, ses travaux portent principalement sur les espaces du public entre dimension matérielle et dimension politique, sur la fabrique architecturale et sur la photographie dans les sciences sociales. Elle a publié Villagexpo. Un collectif horizontal en 2013 (Créaphis éditions) et récemment l'ouvrage issu de sa thèse La visite. Une expérience spatiale aux Presses Universitaires de Rennes (2015). 